\title{
Stress-Induced Gist-Based Memory Processing: A Possible Explanation for Overgeneralization of Fear in Posttraumatic Stress Disorder
}

\author{
Javiera P. Oyarzún ${ }^{1,2 *}$ and Pau A. Packard ${ }^{1 *}$ \\ ${ }^{1}$ Cognition and Brain Plasticity Group, Bellvitge Biomedical Research Institute, L'Hospitalet de Llobregat (Barcelona), Barcelona 08097, Spain, and \\ ${ }^{2}$ Department of Basic Psychology, University of Barcelona, 08035 Barcelona, Spain \\ Review of Qin et al.
}

Stress is a potent modulator of learning and memory. Although stress can selectively enhance memory for emotionally salient information, memory for neutral information within the stressful episode can be impaired (Payne et al., 2006). This impairment is widely thought to be a result of stress-induced catecholaminergic hyperactivity acting on the processes underlying memory formation.

Stimulus-related phasic firing patterns in the midbrain have been proposed to support the hippocampus in forming accurate, detailed memory representations (Lisman and Grace, 2005). Neuroimaging studies have demonstrated significant subsequent memory effects (SMEs), consisting of stronger phasic neural activity for later-remembered than for forgotten items, in hippocampus and midbrain in normal nonstressful contexts (Shohamy and Wagner, 2008), providing evidence of the role of these areas in memory formation. However, how acute stress affects the processing in memory-related neural circuits remains uncertain. As described by Aston-Jones and Cohen (2005), stress-

Received May 14, 2012; revised June 11, 2012; accepted June 14, 2012. We thank Lluís Fuentemilla and Ruth de Diego-Balaguer for their constructive discussions during the development of this article.

*J.P.0. and P.A.P. contributed equally to this work.

Correspondence should be addressed to Pau A. Packard, L'Hospitalet de Llobregat, Barcelona 08097, Spain. E-mail: paupackard@gmail.com. DOI:10.1523/JNEUROSCI.2318-12.2012

Copyright $\odot 2012$ the authors $\quad 0270-6474 / 12 / 329771-02 \$ 15.00 / 0$ induced tonic elevation of background activity in the locus ceruleus-centered norepinephrine (LC-NE) system diminishes such phasic firing patterns. Aston-Jones and Cohen (2005) propose that such a hyperactivation of neuromodulatory systems would produce sensory hypersensitivity, thus generating unselective, generalized responses to stimuli. This would result in a shift toward gist-based memory formation, a type of generalized processing that cuts across discrete experiences (Payne et al., 2002), fostering the encoding of information into categories instead of discrete memory representations.

In a recent paper published in The Journal of Neuroscience, Qin and colleagues (2012) investigated how acute stress affects memory formation, particularly for neutral information. Specifically, they investigated whether stress-induced catecholaminergic activity shifts neural processing into a mode with enhanced tonic activity but reduced phasic responses to stimuli, thus leading to differences in memory formation. For this objective, the authors used event-related functional magnetic resonance imaging (fMRI) during the presentation of complex scenes depicting emotionally neutral activities and experimentally induced acute stress in half of the participants. To verify that participants were stressed, the authors acquired autonomic, endocrine, and psychological measures. In addition, participants went through continuous pupillom- etry during encoding to determine whether stimulus-related phasic pupil responses, which have been shown to parallel LC-NE activity, were altered in relation to memory formation. Participants' memory for the scenes was tested after $24 \mathrm{~h}$, with questions designed to assess memory for the gist or central theme of the presented scenes.

Qin et al. (2012) found that acute stress during the encoding phase led to a more liberal response bias, i.e., an increased tendency to endorse items as having previously been studied, independently of whether they were studied or not. That is, participants under stress made more hits and false alarms (false positives). Moreover, the strength of this bias correlated positively with increased heart rate. Critically, acute stress reduced SMEs during memory formation in the hippocampus and midbrain, and in pupil dilation. This gives support to the view that acute stress can induce a hyperactivation of the catecholaminergic system, which favors the rapid extraction of central thematic information, or gist, and disrupts the phasic signaling in response to stimuli supporting discrete memory formation. This would result in a shift in memory processing that leads to less specificity, less discrete memory representations, a more liberal response bias, and a higher reliance on general (gist-based) aspects.

Qin et al. (2012) point out that a similar mechanism may well account for the 
ample evidence demonstrating the low reliability of eyewitness memories, especially for neutral information. Is it possible that this general mechanism can also explain the memory alterations that typically characterize posttraumatic stress disorder (PTSD) patients? Indeed, one critical hallmark of PTSD is overgeneralization of fear to a wide variety of cues that show only vague similarity with the actual trauma cues. PTSD patients manifest exaggerated fear to cues that do not predict threat even in contexts different from where the traumatic event occurred. Critically, these cues can induce pathological re-experiencing of the original traumatic episode. These cues act as reminders that cause the patient to feel as though the traumatic event was happening again, inducing involuntary intrusive memories (flashbacks) and nightmares. Such distressful episodes can in turn exacerbate other symptoms such as avoidance and hyperarousal. The recurrent association of neutral cues with threat hastens the relapse of anxiety after therapies are successfully accomplished and impedes a total recovery from PTSD.

The formation of traumatic memories has long been investigated and a special interest has been devoted to the role of the hippocampus given the particularities of these memories. PTSD patients can recollect detailed information about the traumatic event (hypermnesia), but much of the retrieved information is fragmented, unspecific, and unreliable. Researchers have established that declarative memory impairments, accompanied by hippocampal abnormalities and dysregulation of the hypothalamic-pituitary-adrenal (HPA) axis, are associated with PTSD.

Diamond and colleagues (2007) proposed a temporal dynamic model of how stress affects memory-related processing in the hippocampus, amygdala, and prefrontal cortex (PFC). They postulated that when stress is maintained, with a tonic activation induced by corticotrophin-releasing hormone (CRH), acetylcholine, dopamine, norepinephrine, and later corticosteroids, the hippocampus enters into a state of desensitization that can lead to neuroplastic impairment over time. However, if hippocampal plasticity or encoding were impaired, it would be difficult to explain why PTSD memories are so strongly consolidated, durable in time, and accessible for explicit retrieval.

Qin and colleagues' (2012) findings suggest that stress-induced hippocampal alteration is instead the result of a different codification strategy for critical situations. In such situations, to efficiently encode survival-related information and avoid irrelevant detail codification, it would be advantageous to acquire information by favoring a categorizing system of information recollection that rapidly extracts the gist of complex stimuli. Thus, it is conceivable that as a consequence of this shift to gistbased memory codification, memory of the traumatic event would be unspecific and fragmented. Importantly, nonselective consolidation of stimuli features, due to a hypervigilant state in PTSD patients, might create a tendency to generalize fear to other cues that share the same gist as those that were present in the traumatic event. This might account for the pathological overgeneralization of fear characteristic of PTSD patients. Therefore, PTSD-related memory alterations may well be explained by an excessive catecholaminergic and HPA axis activity that leads to functional abnormalities in the hippocampus.

The neural model of how stress leads to the unreliable gist-based memories described by Qin and colleagues (2012) may be used to interpret the PTSD-like memory impairment observed recently in mice (Kaouane et al., 2012). To induce PTSD-like memories, Kaouane and colleagues (2012) conditioned mice with different electric foot shock intensities and then administered glucocorticoids to half of them. Mice that were conditioned with the stronger foot shocks and were administered glucocorticoids showed unselective fear responses: while in a safe environment, these mice showed fear responses to cues that did not predict the aversive outcome but had been present in the conditioned context. Moreover, this group of mice responded with strong fear to cues that were never presented before, but that resembled cues presented within the fearful context. Thus, the fear-conditioned mice that were administered glucocorticoids generalized fear to cues that were vaguely related with the traumatic event, as is characteristic of PTSD-like memories.

In summary, Qin and colleagues (2012) provide important hints that may deeply transform our understanding of how memory formation operates during stressful situations. Together with previous studies, the results of this paper prompted our hypothesis that PTSD memory alterations are a consequence of an excessive shift toward gist-memory formation. Our model posits that hyperactivation of stress-sensitive neuromodulatory systems leads to a shift to a memory formation strategy involving unspecific neural firing to stimuli surrounding the traumatic event. This nonselective gistbased memory formation can explain the generalization of fear to discrete cues that were not conditioned, the inability to restrict fear to the appropriate context, and the incapacity to identify correct predictors of threat: all characteristic symptoms of PTSD.

An important question remains to be resolved, given that previous findings regarding hippocampal activity are still not conclusive: Is hippocampal activity reduced/impaired by stress, or is it possible that the hippocampus simply operates under a different encoding strategy in threatful circumstances, as Qin and colleagues (2012) suggest? If the latter is so, it follows that the hippocampus is in fact functioning optimally. To create generalizable fear learning that could be helpful in future similar situations, the hippocampus might relay some of the neutral information processing to neocortical regions (e.g., frontoparietal). PTSD symptoms might be understood then as a result of a conserved and generally adaptive system that, in these patients, generates exaggerated fear in response to numerous incorrect predictors.

\section{References}

Aston-Jones G, Cohen JD (2005) An integrative theory of locus coeruleus norepinephrine function; adaptive gain and optimal performance. Annu Rev Neurosci 28:403-450.

Diamond DM, Campbell AM, Park CR, Halonen J, Zoladz PR (2007) The temporal dynamics model of emotional memory processing: a synthesis on the neurobiological basis of stress-induced amnesia, flashbulb and traumatic memories, and the Yerkes-Dodson law. Neural Plast 2007:60803.

Kaouane N, Porte Y, Vallée M, Brayda-Bruno L, Mons N, Calandreau L, Marighetto A, Piazza PV, Desmedt A (2012) Glucocorticoids can induce PTSD-like memory impairments in mice. Science 335:1510-1513.

Lisman JE, Grace AA (2005) The hippocampalVTA loop: controlling the entry of information into long-term memory. Neuron 46:703-713

Payne JD, Nadel L, Allen JJ, Thomas KG, Jacobs WJ (2002) The effects of experimentally induced stress on false recognition. Memory 10:1-6.

Payne JD, Jackson ED, Ryan L, Hoscheidt S, Jacobs JW, Nadel L (2006) The impact of stress on neutral and emotional aspects of episodic memory. Memory 14:1-16

Qin S, Hermans EJ, van Marle HJ, Fernández G (2012) Understanding low reliability of memories for neutral information encoded under stress: alterations in memory-related activation in the hippocampus and midbrain. J Neurosci 32:4032-4041.

Shohamy D, Wagner AD (2008) Integrating memories in the human brain: hippocampalmidbrain encoding of overlapping events. Neuron 60:378-389. 